\title{
RETROSPECTIVE ANALYSIS OF LSIS VERSUS PHARMACOLOGICAL MANAGEMENT IN ACUTE ANAL FISSURE- A STUDY IN ZONAL SERVICE HOSPITAL
}

\author{
Bharat K. Jani ${ }^{1}$, Hemant S. Nagar ${ }^{2}$
}

${ }_{1}$ Classified Specialist Surgery, Department of General Surgery, INHS, Dhanvantari, Port Blair, A and N Island, India.

2 Professor in Surgery and Consulting Paediatric Surgeon, Department of General Surgery, TS Misra Medical College, Amausi, Lucknow, Uttar Pradesh, India.

\section{ABSTRACT}

\section{BACKGROUND}

Acute anal fissure is a quite common and extremely painful condition of anal canal. The prevalence of anal fissures has changed due to change in food habits in the Indian subcontinent. Medical management is a recent trend in the treatment of anal fissure; however, the parameters for measurement of outcome are result-based and not measuring quality of life post treatment. Here, we have conducted a retrospective study of our patients, which were managed by various medical methods and lateral subcutaneous internal sphincterotomy. The results of the study were documented and studied retrospectively. The results were analysed on various parameters of symptomatic relief of pain, after different periods of time and improved comfort during and after treatment. The incidence of anal fissure in males and females were also studied. Recent studies and clinical work are advocating medical management of acute anal fissure citing reasons of early recovery and avoiding unnecessary admissions. However, it seems a very short-sighted development, because either the cases which had recurrence may not have reported back or it may be short term solution without considering long-term implications.

The aim of the study was to evaluate role of Lateral Subcutaneous Internal Anal Sphincterotomy (LSIAS) vis-a-vis conservative management in cases of acute anal fissure in terms of clinical parameters.

\section{MATERIALS AND METHODS}

It is a descriptive study of results of patients in a service-hospital surgical-OPD and in-patient cases. Patients were administered treatment and results were analysed at the time of discharge. Data of only those patients were used who could be followed up for next 6 months.

\section{RESULTS}

In our clinical study, it was found that surgical intervention in the initial and first encounter has better overall response as compared to conservative management over a period of time.

\section{CONCLUSION}

With this study it can be safely concluded that in an acute anal fissure, surgical intervention in the first setting has maximum chances of early recovery, reduced loss of man hours, comparatively better post-treatment period and importantly reduced chances of progress to chronic anal fissure. It does not absolutely rule out the role of conservative management by using GTN and CCBs in addition to the traditional conservative supportive management; however, it needs strict compliance with the treatment protocol, sudden change in dietary preferences and a cooperative patient. It is also understood that pain and sense of constant discomfort in anal canal is more disturbing and an important parameter than others while considering response to treatment outcome.

\section{KEY WORDS}

Sphincter Spasm, Internal Anal Sphincter, Lateral Subcutaneous Internal Anal Sphincterotomy (LSIAS).

HOW TO CITE THIS ARTICLE: Jani BK, Nagar HS. Retrospective analysis of LSIS versus pharmacological management in acute anal fissure- a study in zonal service hospital. J. Evolution Med. Dent. Sci. 2018;7(41):4473-4477, DOI: 10.14260/jemds/2018/997

\section{BACKGROUND}

Regular bowel motions are one of the most satisfying activities and an important indicator of general health and well-being of an individual. Anal canal is a complex structure with finely arranged muscles with voluntary and involuntary innervation, richly innervated and a site for porto-systemic anastomosis. ${ }^{1}$

'Financial or Other Competing Interest': None.

Submission 25-08-2018, Peer Review 22-09-2018,

Acceptance 28-09-2018, Published 08-10-2018.

Corresponding Author:

Dr. Bharat K. Jani,

INHS, Dhanvantari, Minnie Bay,

Port Blair, A and N Islands-744103,

India.

E-mail: drbharatjani@gmail.com

DOI: $10.14260 /$ jemds $/ 2018 / 997$

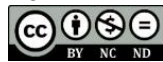

Acute anal fissure is caused due to sudden traumatic injury to lower anal mucosa causing extremely painful acute spasm of anal canal. This is mostly precipitated by hard stools and voluntary stressful defecation against closed internal sphincter. The anal sphincter spasm is perceived by patient as presence of hard stools and in an effort to complete the defecation process he/she tries to do a voluntary effort against a closed sphincter subsequently injuring the delicate anal mucosa.

Despite being a common disorder the management has by and large remained conservative and with the change in lifestyle and fast-moving world, the research work is also more inclined to get less interventional and more conservative approach. Management by pharmacological relaxation of the internal anal sphincter with NTG, calcium channel blockers, botulinum toxins and others likewise is well published. Surgical management of anal fissure involves 
few established procedures like LSIAS, fissurectomy, fissurectomy with V-Y anoplasty. Theoretical principles of conservative management appears more promising on papers only. Moreover due to reputation of anorectal procedures in market, despite these established procedures and excellent post-op results clients prefer conservative methods. However, fissurectomy involves longer hospital stay and preferably done in chronic anal fissures. V-Y anoplasty is technically more demanding and results may have conflict of interest. Hence, LSIAS is a preferred method by author.

\section{Review of Literature}

\section{Glyceryl Trinitrate/ Chemistry}

Organic nitrates are polyol esters of nitric acid, whereas organic nitrites are esters of nitrous acid. Nitrate esters $\left(\mathrm{CONO}_{2}\right)$ and nitrite esters (CONO) are characterised by a sequence of carbon-oxygen-nitrogen, whereas nitro compounds possess carbon-nitrogen bonds $\left(\mathrm{CNO}_{2}\right)$. Thus, Glyceryl trinitrate is not a nitro compound, and it is erroneously called nitroglycerin; however, this nomenclature is both widespread and official. The efficacy of GTN is limited by headaches, dizziness and tachyphylaxis. ${ }^{2}$ It achieves the reduction in Mean Rectal Pressure (MRP) for 15 - 90 minutes. ${ }^{3}$ These dynamics makes the application schedule quite frequent; however, it causes headache which is more debilitating.

Patients with chronic fissures should be started on the acute fissure regimen, but are typically also started on other therapies simultaneously including nitroglycerin or isosorbide dinitrate, theoretically producing "reversible chemical sphincterotomy." For nitroglycerin the limiting side effects are headaches and tachyphylaxis, which can be reduced by instructing the patient to rest lying down while applying the ointment. The topical application of diltiazem (2\%), a calcium channel blocker, produces fewer side effects and similar efficacy as nitroglycerin. Fissure healing can be anticipated in about $70 \%$ of patients with chronic fissures using nitroglycerin or diltiazem. ${ }^{4}$ It has been observed that chronic anal fissure is ischaemic in origin due to poor blood supply and spasm of internal anal sphincter. However, during the course of therapy, strict dietary restrictions to smoothen the stool are necessary. Headache during therapy is a major concern with the incidence as high as $20-100 \%$. Though the application of GTN has a high healing rate, it also has a high recurrence rate. ${ }^{3}$

\section{Calcium Channel Blocker (Nifedipine)}

In both smooth muscle and cardiac myocytes, $\mathrm{Ca}^{2+}$ is a trigger for contraction, albeit by different mechanisms. $\mathrm{Ca}^{2+}$ channel antagonists, also called $\mathrm{Ca}^{2+}$ entry blockers inhibit $\mathrm{Ca}^{2+}$ channel function. Oral Nifedipine is an L-type calcium channel antagonist. L-type calcium channels are the principle calcium channels in the GI smooth muscles. In the treatment of anal fissures, $20 \mathrm{mg}$ of Nifedipine is applied locally twice daily. Nifedipine is found effective in relieving the sphincter spasm. It is known for achieving increase in the local blood supply by an independent mechanism. This allows faster healing. It is however supposed to cause reversible internal anal sphincterolysis. Most of these drugs have a short duration of action and need to be administered 2 - 3 times daily. Similarly, side effects like headache, palpitations, flushing, dizziness, colicky abdominal pain, ankle oedema, reduced taste and smell, nausea and diplopia have been reported. 4

\section{Lateral Subcutaneous Internal Anal Sphincterotomy (LSIAS)}

This procedure may be performed with regional or general anaesthesia. The intersphincteric groove and internal sphincter are identified and delivered through a submucosal wound. A deliberate cut is made at either 3 or 9 0'clock position followed by anal packing. A small tampon is placed in the anal canal to prevent haematoma. Results from the literature do not support better healing rates for the open technique and generally describe a greater frequency of complication; however, open sphincterotomy is more appealing from a training standpoint because the internal sphincter can be directly visualised and the extent of transection more readily quantitated, early and late complications can occur after lateral internal sphincterotomy including urinary retention, bleeding and abscess or fistula formation as well as seepage and rarely incontinence. ${ }^{2} \mathrm{~A}$ healed fissure is defined as epithelialisation achieved on 12th week of therapy not associated with pain, bleeding or other local symptoms. Complete absence of pain was considered as symptomatic pain relief, while complete epithelialisation of fissure was labelled as healed fissure. ${ }^{5}$

\section{MATERIALS AND METHODS}

It is a descriptive study of results of patients in a service hospital surgical OPD and in-patient cases. Patients were administered treatment and results were analysed at the time of discharge. Data of only those patients were used who could be followed up for next 6 months.

The inclusion criteria was presence of acute anal fissure during clinical examination, a willing and cooperative patient, patients who came for regular follow-up with reliable inputs. The study design has excluded all those patients who were having comorbidities (diabetes, hypothyroidism, pulmonary Koch's and chronic lifestyle disorders) and patients who were lost for follow-up.

The patients were regularly followed up in OPD. The symptomatic relief was measured on the basis of pain relief during defecation, post defecation sense of spasm, difficulty in doing sitting work, feeling of complete defecation (on a scale of $1-10)$.

The Schedule of Conservative Management was followed as shown in Table No. 1

\begin{tabular}{|c|c|c|c|c|}
\hline Sl. No. & Method of Treatment & Schedule of Treatment & Method of Application & Dietary Advice \\
\hline \multirow{3}{*}{1} & \multirow{3}{*}{ Traditional } & Sitz bath & Three times a day & \multirow{3}{*}{$\begin{array}{l}\text { (a) Avoid spicy, low } \\
\text { fibre food } \\
\text { (b) Plenty of oral fluids }\end{array}$} \\
\hline & & Stool softeners & Liquid paraffin $20 \mathrm{~mL} \mathrm{HS}$ & \\
\hline & & Bulk forming agents & Isabgula Husk- one sachet at night & \\
\hline 2 & NTG & $\begin{array}{c}0.2 \% \text { GTN ointment applied twice to } \\
\text { the anoderm for } 6 \text { weeks in addition } \\
\text { to traditional treatment }\end{array}$ & $\begin{array}{l}\text { Twice a day application on anoderm } \\
\text { and anal mucosa after defecation }\end{array}$ & $\begin{array}{c}\text { (c) Do not strain while } \\
\text { passing stools } \\
\text { (d) Keep perineal area }\end{array}$ \\
\hline
\end{tabular}




\begin{tabular}{|c|c|c|c|c|}
\hline 3 & Nifedipine & $\begin{array}{c}20 \text { mg of Nifedipine for six weeks } \\
\text { addition to traditional treatment }\end{array}$ & $\begin{array}{c}\text { Twice a day local application on } \\
\text { anoderm and anal mucosa after } \\
\text { defecation }\end{array}$ & dry and clean \\
\hline \multicolumn{3}{|c|}{ Table 1 } \\
\hline
\end{tabular}

\section{Lateral Subcutaneous Internal Anal Sphincterotomy (LSIAS)}

As per institution protocol, preoperative workup was done for all those who underwent surgical procedure. All surgeries were done under spinal anaesthesia. Bowel preparation was not done. Only liquid diet for one day and fasting past midnight before surgery and IV antibiotics on the morning of surgery was administered. ${ }^{4}$

\section{Steps of Surgery}

1. On operation table, per rectal examination and proctoscopy under anaesthesia was done to rule out any underlying hidden pathology and anal stenosis.

2. Park's anal retractor was inserted and internal sphincter was stretched and made easily palpable. Cautery was used to give incision and dissect through groove to ensure bloodless field. A small incision was then made on the lateral aspect of anal canal at 3 and 9 O'clock position. IAS was delivered in the wound using artery forceps, sphincter was identified by its white fibres, confirmed and divided with cautery and cut deliberately using coagulation current.

3. The main wound was washed thoroughly, haemostasis confirmed and left open for secondary healing. Anal pack with medicated paraffin gauze applied, which was removed the same evening.

4. Post-operatively, IV antibiotics for one day and normal diet from next day were begun. Patients were discharged next day with advice of local wound care.

5. Patients in both the groups were prescribed to take stool softeners and Isabgula Husk and do sitz bath three times a day for next 4 weeks. Patients were followed up in outpatient department at regular intervals.

\section{Follow-Up}

All the patients were followed up at the end of month, $3^{\text {rd }}$ month and after 6 months. In addition to the relief of symptoms they were interviewed for requirement of taking rest from work due to symptoms of fissure, relief from symptom of constant discomfort post defecation, sense of constant tightness and spasm in anal canal and status of general happiness as far as effect of treatment is concerned. A high-fibre diet was encouraged. All the patients were followed up in OPD with emphasis on follow-up of pain are standard period of hospitalisation for 3 days for all surgical patients as per the institutional protocol. Hence, anything more than 3 days was given significance and considered in the study.

Pain and perianal discomfort was the main symptom concentrated upon. In addition to this adequate relaxation during defecation, post defecation pain and sense of constant spasm in anal canal was also asked for. A composite grading for all the symptoms was considered, while evaluating relief of symptoms. Clinical per rectal examination and presence of anal tone, pain during examination was correlated with the symptoms.

Effects of treatment were like headache, dizziness, flush, rash and incontinence. Healing of acute anal fissure was set as the primary target of our study. However, clinical examination of the fissure and evaluation of other parameters is also done for complete analysis. All the results are compiled in tabulated format and statistically analysed for comparison of outcome. Few patients were admitted for more than 3 days due to administrative reason (convenience of sitz bath in field area for service personnel).

This is a retrospective analysis, hence only those cases which are included in the study were selected because they were satisfying all inclusion and exclusion criteria and could be measured on all parameters of study. The evaluation was simply done on the percentage response proportional to the sample size. Percentage of each calculated out of those who underwent procedure A were considered in the study. Those who claim to have pain relief also had different levels of relief, hence pain relief with full comfort life is taken into consideration.

\section{RESULTS}

More number of adult males underwent surgical intervention. This may be due to the service requirements, field locations and inconvenience to carry out conservative management. Females are found to have more inclination towards conservative management, probably due to apprehension of hospitalisation and worries of leaving kids at home (as they come out during interviews in OPD). However, those who underwent procedure A for surgical intervention were more comfortable after discharge, as they could conveniently carry out their daily work due to quick relief from the symptoms. All the results are placed in Table No. 2.

\begin{tabular}{|c|c|c|c|c|c|}
\hline \multirow[t]{2}{*}{ Sl. No. } & \multirow[t]{2}{*}{ Parameters/ Procedures } & \multirow[t]{2}{*}{ LSIS 80/150 } & \multicolumn{3}{|c|}{ Conservative $(\mathbf{7 0} / \mathbf{1 5 0})$} \\
\hline & & & $\begin{array}{c}\text { Supportive } \\
\mathbf{3 0} / 70 \\
\end{array}$ & $\begin{array}{c}\text { Nitroglycerin } \\
23 / 70 \\
\end{array}$ & $\begin{array}{c}\text { CCB } \\
17 / 70 \\
\end{array}$ \\
\hline 1 & Pain relief on day 1 & 80 & $10(30 \%)$ & $12(52.17 \%)$ & $9(52.94 \%)$ \\
\hline 2 & Hospitalisation for more than 3 days & $10(12.5 \%)$ & 0 & 0 & 0 \\
\hline 3 & Symptomatic after 1 month & $3(3.75 \%)$ & $20(66.66 \%)$ & $10(43.47 \%)$ & $11(64.7 \%)$ \\
\hline 4 & Symptomatic after 3 months & $2(2.5 \%)$ & $18(60 \%)$ & $9(39.13 \%)$ & $10(58.82 \%)$ \\
\hline 5 & Recurrence after 6 months & $5(6.25 \%)$ & $20(66.66 \%)$ & $10(43.47 \%)$ & $9(52.94 \%)$ \\
\hline 6 & Progression to chronic & $2(2.5 \%)$ & $22(73.33 \%)$ & $10(43.47 \%)$ & $9(52.94 \%)$ \\
\hline 7 & \begin{tabular}{|c|} 
Average loss of work days -3 \\
\end{tabular} & $3(3.75 \%)$ & $3(10 \%)$ & $3(13.04 \%)$ & $3(17.64 \%)$ \\
\hline 8 & Discomfort level during and after treatment $\left({ }^{*}\right.$-Scale & $9(11.25 \%)$ & $6(20 \%)$ & $8(34.78 \%)$ & $4(23.52 \%)$ \\
\hline
\end{tabular}




\begin{tabular}{|c|c|c|c|c|}
\hline & of 1-10; 1- poor, 10- most comfortable) & & & \\
\hline & $1-3$ & 5 & 3 & 5 \\
\hline \\
\hline
\end{tabular}

Table 2. Results of Retrospective Analysis of Acute versus Conservative Management

Paediatric patients did not undergo any surgical procedure, as conservative management could relieve the symptoms. Amongst the patients who were managed conservatively, more than $50 \%$ of patients remained symptomatic till a month with pain and post defecation residual spasm except in case of GTN (39.13\%). However, GTN-induced headache was a major complaint by patient.

\section{DISCUSSION}

Acute anal fissure is a fairly common entity found amongst Indian population and the trend is increasing recently due to changed dietary habits. It is also seen more amongst postnatal females, executive professionals and anxious personalities. Disturbed bowel habits are taken as a stigma by a large number of people. In addition to the dietary preferences and other health conditions, acute anal fissure is also related with individual personality. ${ }^{6}$

Amongst those who remained symptomatic at the end of 4 weeks continued to complain till end of 12 weeks, which may indicate the difference in limit of relaxation of internal sphincter by chemical method in different patients. Almost equal number of patients presented again after 6 months and found to have features of chronic transformation of anal fissure. The period of hospitalisation includes a day prior for anaesthesia workup.

When stool enters the rectum it distends and the involuntary internal anal sphincter (which is normally contracted) relaxes, while the external anal sphincter (under voluntary control) remains closed. This process is called the Rectoanal Inhibitory Reflex (RAIR). ${ }^{7}$ The external sphincter relaxes when there is an 'appropriate time' for defecation. However, if during this 'appropriate time' the involuntary sphincter is contracted (due to pain/ irritation due to physical/ chemical trauma/ food), the individual strains and tries to defecate against closed sphincter. This results in tear in acute anal mucosa and initiates the vicious cycle.

It is also important to note that though the anal canal is richly innervated, but is not equally nourished. Especially, during long-standing spasm, the anal mucosa is deprived of vascular nourishment which further delays healing of the fissure. Adequate relief in internal sphincter spasm is a key factor in early resolution of symptoms. Many of the times, food with high spicy content causes irritation to distal anal mucosa and causes spasm of internal sphincter as a response to local irritation. This also initiates the vicious cycle as described above.

This explains occurrence of fissure in patients with no history of constipation at all. ${ }^{8}$ In such patients with underlying cause being irritation due to spicy food medical management is likely to fail, as the pharmacological sphincterotomy does not give complete relaxation with sudden change in dietary schedule may not be possible at all. A recent study by 'Sajith SM et al' has revealed good response to conservative management using CCBs; however, it did not include the feeling of constant tightness in the anal canal which is an indicator of persistent anal spasm, which is a strong predisposing factor for chronicity of fissure. ${ }^{9}$

It is also to be noted that feeling of tightness and incomplete evacuation is the major concern of all patients. Symptomatic patients most stressfully mention pain as major symptom pre- and post-treatment. Repeated pain during each defecation and an individual further becomes apprehensive to go for defecation. Conservative management by traditional conservative management has not reduced pain and feeling of constant tightness in anal canal. Though GTN and CCBs has reduced suffering of patients in terms of post defecation pain, they could not reduce the sense of tightness in the anal canal and low-grade long-term pain. Immediate pain relief as a strong positive point of LSIS, which starts on immediate postop period as internal sphincter is the main culprit causing pain. An overexcited IAS gives sense of constant tightness in anal canal and post defecation pain. Hence, surgical sphincterotomy is always superior to medical methods. ${ }^{10}$

Almost equal number of patients remained symptomatic after 1 and 3 months. These are likely to be those individuals whose anal sphincter has reached to a threshold of relaxation and medical methods are no longer going to reduce the spasm. These patients are likely to go in chronic mode and will invariably be benefitted by the surgical sphincterotomy.

The mechanism and pathophysiology of formation of acute anal fissure and transformation into a chronic anal fissure is a prolonged process, which involves repeated subclinical insults to anal sphincter mechanism. The repeated insults of the anal sphincter lands it into a malfunctioning valve. With this analysis it is understood that the conservative management using GTN and CCBs is also a causative agent for transformation of acute into chronic fissure, as the sphincter fails to relax completely. Recurrence of symptoms after six months is significantly high in patients with conservative management. This is probably because of persistence of causative agent or inadequate relaxation of IAS.

One important aspect in the outcome is loss of work days and quality of life during and post treatment. Though, there is an absolute loss of three workdays in LSIS, the quality of life is much better post treatment due to absolute pain free period. There has been no incidence of anal incontinence as LSIS is done at 3 and 9 O'clock position, which preserves the RAIR. In the conservative management the patient has to remain in pain for a long duration of time, do a regular and strict adherence to supportive and dietary measures and be highly compliant to treatment protocol which may not be possible for working population.

\section{CONCLUSION}

With this study, it can be safely concluded that in an acute anal fissure surgical intervention in the first setting has maximum chances of early recovery, reduced loss of man hours, comparatively better post treatment period and importantly reduced chances of progression to chronic anal fissure. It does not absolutely rule out the role of conservative management by using GTN and CCBs in addition to the 
traditional conservative supportive management; however, it needs strict compliance with the treatment protocol, sudden change in dietary preferences and a cooperative patient. It is also understood that pain and sense of constant discomfort in anal canal is more disturbing and an important parameter than others, while considering response to treatment outcome.

\section{REFERENCES}

[1] Gordon PH, Nivatvongs S. Principles and practice of surgery for the colon, rectum, and anus. Part I: $3^{\text {rd }}$ edn. Essential Considerations, Informa Healthcare USA, Inc. CRC Press 2007: p. 12.

[2] Michel T. Treatment of myocardial ischemia. Chapter -31. Goodman Gillman's the pharmacological basis of therapeutics. 11th edn. McGraw-Hill Medical Publishing Division 2006: p. 3-4.

[3] Bhardwaj R, Parker MC. Modern perspectives in the treatment of chronic anal fissures. Ann R Coll Surg Engl 2007;89(5):472-8.

[4] Lindsey I, Jones OM, Cunningham C, et al. Chronic anal fissure. Br J Surg 2004;91(3):270-9.
[5] Nelson H. Townsend: Sabiston textbook of surgery. Section 10, Chapter - 49. Benign anorectal diseases (e-book). $7^{\text {th }}$ edn. Elsevier International Edition, eedn. 2004: p. 1494.

[6] Garcea G, Sutton C, Mansoori S, et al. Results following conservative lateral sphincterotomy for the treatment of chronic anal fissures. Colorectal Dis 2004;6:210-11.

[7] Hosseinzadeh ST, Poorsaadati S, Radkani B, et al. Psychological disorders in patients with chronic constipation. Gastroenterol Hepatol Bed Bench 2011;4(3):159-63.

[8] Ehrenpreis ED. Remedica explained series, anal and rectal diseases explained. Remedica Publishing 2003.

[9] Siddiqi S, Vijay V, Mahendran R, et al. Pruritus Ani. The Annals of The Royal College of Surgeons of England 2008;90(6):457-63.

[10] Babu SSM, Gupta R, Singh LS. Effectiveness of conservative management of acute fissure in ano: a prospective clinical study of 165 patients. International Surgery Journal 2017;4(9):3028-33. http://www.ijsurgery.com 